\title{
Discrete Hedging Under Piecewise Linear Risk Minimization
}

\author{
Thomas F. Coleman`, Yuying $\mathrm{Li}^{\star}$, Maria-Cristina Patron ${ }^{\star \star}$
}

* Cornell Theory Center and Department of Computer Science, Cornell University,

Ithaca, NY 14853, USA, email contact: coleman@tc.cornell.edu

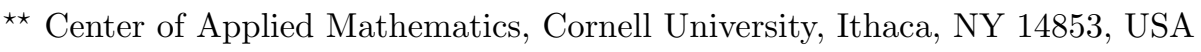

December 20, 2002 


\begin{abstract}
In an incomplete market it is usually impossible to eliminate the intrinsic risk of an option. In this case quadratic risk-minimization is often used to determine a hedging strategy. However, it may be more natural to use piecewise linear risk-minimization. We investigate hedging strategies using piecewise linear risk-minimization. We illustrate that this criterion for risk-minimization may lead to smaller expected total hedging cost and significantly different, possibly more desirable, hedging strategies from those of quadratic risk minimization. The distributions of the total hedging cost and risk show that hedging strategies obtained by piecewise linear risk-minimization have a larger probability of small cost and risk, though they also have a very small probability of larger cost and risk. Comparative numerical results are provided. We also prove that the value processes of these hedging strategies satisfy put-call parity.
\end{abstract}

Keywords: incomplete market, discrete hedging, piecewise linear risk-minimization, quadratic riskminimization, cumulative cost, incremental risk 


\section{Introduction}

Investors try to reduce the sensitivity of their portfolios to the fluctuations of the market by hedging. In particular, in option hedging, one tries to construct a trading strategy that replicates the option payoff and does not require any inflow or outflow of capital other than initial costs. In the Black-Scholes framework, the option can be replicated by using only the underlying asset and a bond. However, the investor's position is only instantaneously riskfree and therefore, it must be adjusted continuously. In practice, a natural problem that occurs is the impossibility of hedging continuously in time coupled with the need to hedge as little as possible due to the impact of transaction costs. If only discrete hedging times are allowed, achieving a risk-free position at each time is no longer appropriate since this instantaneous hedging will not last till the next rebalancing time. Moreover, the market becomes incomplete and the Black-Scholes framework cannot be applied. Under these conditions, there is much uncertainty regarding the choice of an optimal hedging strategy and in defining the fair price of an option. It is not possible to totally hedge the intrinsic risk carried by options that cannot be exactly replicated. An "optimal" hedging strategy can be chosen to minimize a particular measure of this risk.

Different criteria for quadratic risk minimization can be found in the literature. We mention, for example, Föllmer, and Schweizer (1989), Schäl (1994), Schweizer (1995, 2001), Mercurio, and Vorst (1996), Heath, Platen, and Schweizer (2001a, 2001b). We only briefly describe them here, but they are presented in more detail in Section 2.

Suppose we want to hedge an option and we only have a finite number of hedging times: $t_{0}, t_{1}, \ldots, t_{M}$. Suppose also that the discounted underlying asset price is a square integrable process on a probability space $(\Omega, \mathcal{F}, P)$, with filtration $\left(\mathcal{F}_{k}\right)_{k=0,1, \ldots, M}$. Denote by $C_{k}$ the cumulative cost of the hedging strategy up to time $k$ (this includes the initial cost for setting up the hedging portfolio and the cost for rebalancing it at the hedging times $\left.t_{0}, \ldots, t_{k}\right)$.

Currently, there are two main quadratic hedging approaches for choosing an optimal strategy. One possibility is to control the total risk by minimizing the $L^{2}$-norm $E\left(\left(C_{M}-\right.\right.$ $\left.C_{0}\right)^{2}$ ), where $E(\cdot)$ denotes the expected value with respect to the probability measure $P$. This is the total risk-minimization criterion. An optimal strategy for this criterion is selffinancing, that is, its cumulative cost process is constant. However, a total risk-minimization strategy may not exist in general. The additional assumption that the discounted underlying asset price has a bounded mean-variance tradeoff is required. In this case, there exists an explicit strategy. The existence and the uniqueness of a total risk-minimizing strategy have been extensively studied by Schweizer (1995).

Another possibility is to control the local incremental risk, by minimizing $E\left(\left(C_{k+1}-\right.\right.$ $\left.C_{k}\right)^{2} \mid \mathcal{F}_{k}$ ) for all $0 \leq k \leq M-1$. This is the local quadratic risk-minimizing criterion. The same assumption that the discounted underlying asset price has a bounded meanvariance tradeoff is sufficient for the existence of an explicit local risk-minimizing strategy (see Schäl 1994). This strategy is no longer self-financing, but it is mean-self-financing, i.e., the cumulative cost process is a martingale. In general, the initial costs for the local riskminimizing and total risk-minimizing strategies are different. As Schäl noticed, the initial costs agree in the case when the discounted underlying asset price has a deterministic meanvariance tradeoff. He then suggests the interpretation of this initial cost as a fair hedging price for the option. However, as shown by Schweizer (1995), this is not always appropriate.

In order to justify the optimal hedging strategy of the quadratic risk minimization and to ascertain the fair value of the option, it is important to analyze the dependence of the optimal hedging strategy on the choice of the quadratic risk measure. We remark that the optimal hedging strategy hinges on the subjective criterion for measuring the risk. 
Under the Black-Scholes assumptions, an option can be hedged with a bond and the underlying asset, with no risk, i.e., zero incremental in or out cashflow for rebalancing. When rebalancing can only be done at discrete times, a natural optimal hedging strategy is the one which minimizes the expected magnitude of the incremental cashflow; this leads to the optimization problems, minimize $E\left(\left|C_{k+1}-C_{k}\right| \mid \mathcal{F}_{k}\right)$ or minimize $E\left(\left|C_{M}-C_{0}\right|\right)$, respectively.

Another argument in favor of the piecewise linear risk measure is that the costs of rebalancing usually have a linear form. Since $C_{k+1}-C_{k}$ represents the change in value of the portfolio, minimizing $E\left(\left|C_{k+1}-C_{k}\right| \mid \mathcal{F}_{k}\right)$ seems more likely to yield smaller rebalancing costs.

In addition, minimizing the piecewise linear risk, $E\left(\left|C_{k+1}-C_{k}\right| \mid \mathcal{F}_{k}\right)$, and minimizing the quadratic risk, $E\left(\left(C_{k+1}-C_{k}\right)^{2} \mid \mathcal{F}_{k}\right)$, can lead to significantly different solutions. Assume that $p_{k}(S)$ is the conditional density function of the underlying price at time $t_{k+1}$. Minimizing $E\left(\left(C_{k+1}-C_{k}\right)^{2} \mid \mathcal{F}_{k}\right)$ emphasizes more on reducing the largest value of $\sqrt{p_{k}(S)}\left|C_{k+1}-C_{k}\right|$, whereas minimizing $E\left(\left|C_{k+1}-C_{k}\right| \mid \mathcal{F}_{k}\right)$ attempts to reduce the density weighted incremental cashflow, $p_{k}(S)\left|C_{k+1}-C_{k}\right|$, for each underlying value $S$, equally. This suggests that minimizing $E\left(\left|C_{k+1}-C_{k}\right| \mid \mathcal{F}_{k}\right)$ is more likely to yield a smaller incremental cashflow (see subsequent example and numerical results). Moreover, focusing on reducing the largest value of $\sqrt{p_{k}(S)}\left|C_{k+1}-C_{k}\right|$ does not imply minimizing the extreme incremental cost $\left|C_{k+1}-C_{k}\right|$ if the density of such an extreme value is sufficiently small.

To illustrate the above discussion in more detail, consider the following comparison between the piecewise linear risk-minimization with respect to the local risk measure $E\left(\mid C_{k+1}-\right.$ $\left.C_{k}|| \mathcal{F}_{k}\right)$, and the quadratic risk-minimization with respect to $E\left(\left(C_{k+1}-C_{k}\right)^{2} \mid \mathcal{F}_{k}\right)$. Suppose the price of the underlying asset follows the stochastic differential equation:

$$
\frac{d S_{t}}{S_{t}}=\mu d t+\sigma d Z_{t}
$$

where $Z_{t}$ is a Wiener process. Let the initial value of the asset $S_{0}=100$, the instantaneous expected return $\mu=.2$, the volatility $\sigma=.2$ and the riskless rate of return $r=.1$. Suppose we want to statically hedge a deep in-the-money and a deep out-of-money put option with maturity $T=1$; we only have one hedging opportunity, at time 0 . At the maturity $T$ we compare the payoff of the options with the hedging portfolio values of the strategies obtained by the piecewise linear and quadratic local risk-minimization. The payoff and the hedging portfolio values at time $T$ are multiplied by the density function of the asset price and are discounted to time 0 . The first plot in Figure 1 shows the weighted payoff and the weighted values of the hedging portfolios at the maturity $T$ for the in-the-money put option. The second plot presents the corresponding data for the out-of-money put option. 

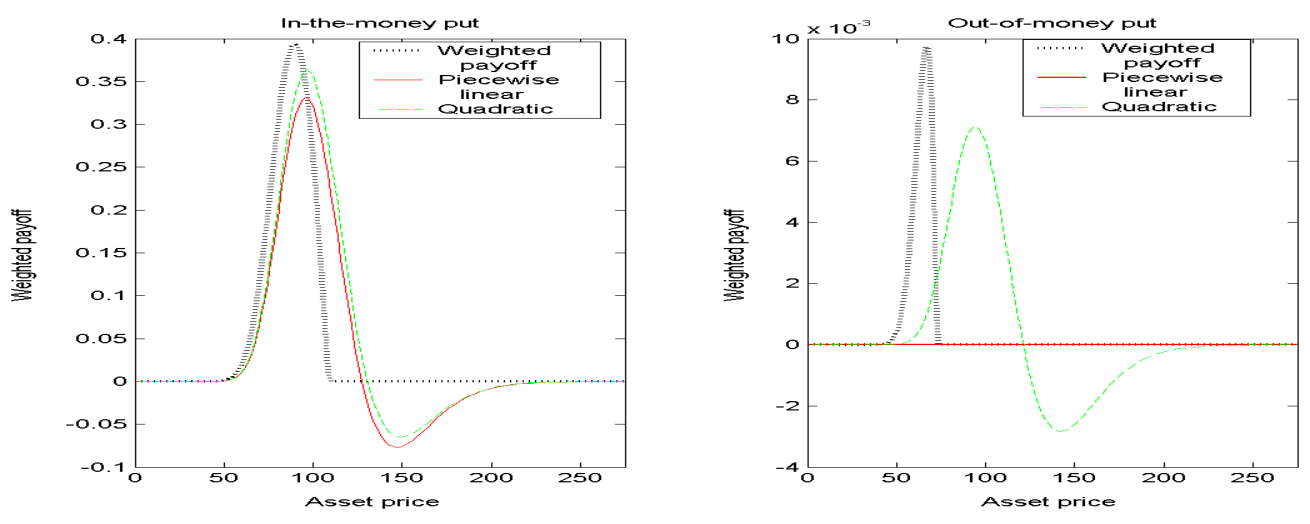

Figure 1: Best fitting of the option payoff

In the case of the in-the-money put option, the weighted payoff, closer to normal, is much easier to fit. We remark that in this case both criteria generate similar plots of the hedging strategy values and these fit relatively well the option payoff. However, the weighted payoff for the out-of-money put option seems more difficult to match. Despite the small values (of order $10^{-3}$ ), it is important to note that the relative differences between the weighted payoff and the weighted values of the hedging portfolios are large. (The cost of an out-of-money put is much smaller than the cost of the in-the-money put.) We have illustrated the hedging of only one out-of-money put option; if we want to hedge 100 put options identical to the one considered, the absolute differences between the weighted payoff and the weighted hedging portfolio values will also be significant. The hedging styles of the two strategies are very different. The $L^{2}$-norm (i.e., quadratic) attempts to penalize large residuals excessively and this actually leads to a worse fit under most scenarios. Indeed, the probability that the put option expires out of money is very large, around .97, but the $L^{2}$ hedging strategy either over or under replicates the option payoff. On the other hand, the $L^{1}$ strategy hedges exactly the option payoff when it expires out of money. Suppose we short the out-of-money put option. At the maturity of the option, our possible losses are never greater than the strike price. Assume now that we want to hedge our position by buying the $L^{2}$ hedging strategy. We can see from the figure that, by excessively trying to reduce the risk in the unlikely event that the option expires in the money, the $L^{2}$ strategy actually introduces the very small probability of unlimited losses. This is not the case if we try to hedge the short position using the $L^{1}$ strategy.

Unfortunately, there are no known analytic expressions for the optimal hedging strategies in the case of the risk measures $E\left(\left|C_{M}-C_{0}\right|\right)$ and $E\left(\left|C_{k+1}-C_{k}\right| \mid \mathcal{F}_{k}\right)$. In this paper we concentrate on describing alternative hedging strategies for local risk-minimization. We compare the effectiveness of the hedging strategies based on piecewise linear risk minimization to those based on quadratic risk minimization. We first illustrate that, by generating synthetic paths for the asset price, the piecewise linear risk minimization may lead to smaller average total hedging cost and risk. We then confirm these results by computing the expected total cost and risk in the binomial view of the asset price. Finally we provide numerical results which emphasize the fact that the optimal hedging strategies with respect to piecewise linear risk-minimization are significantly different, possibly more desirable, having a larger probability of small cost and risk, although a very small probability of larger cost and risk than the traditional strategies.

In Section 2 of the paper we review the criteria for quadratic risk-minimization. Al- 
ternative criteria based on piecewise linear risk-minimization are described in Section 3. Section 4 illustrates numerically the differences between the quadratic and the piecewise linear local risk-minimizing strategies. In Section 5 we show that the value processes of the hedging strategies satisfy put-call parity and and we conclude in Section 6 .

\section{Quadratic risk-minimization}

Consider a financial market where a risky asset (called stock) and a risk-free asset (called bond) are traded. Let $T>0$ and let $0=t_{0}<t_{1}<\ldots<t_{M}=T$ be discrete hedging dates. Suppose $(\Omega, \mathcal{F}, P)$ is a filtered probability space with filtration $\left(\mathcal{F}_{k}\right)_{k=0,1, \ldots, M}$, where $\mathcal{F}_{k}$ corresponds to the hedging time $t_{k}$ and w.l.o.g. $\mathcal{F}_{0}=\{\emptyset, \Omega\}$ is trivial. Assume the stock price follows a stochastic process $S=\left(S_{k}\right)_{k=0,1, \ldots, M}$, with $S_{k}$ being $\mathcal{F}_{k}$-measurable for all $0 \leq k \leq M$. We can set the bond price $B \equiv 1$ by assuming the discounted stock price process $X=\left(X_{k}\right)_{k=0,1, \ldots, M}$, where $X_{k}=\frac{S_{k}}{B_{k}}, \forall 0 \leq k \leq M$.

Suppose we want to hedge a European option with maturity $T$ whose payoff is given by a $\mathcal{F}_{M}$-measurable random variable $H$. For example, in the case of a European put with maturity $T$ and discounted strike price $K$, we have $H=\left(K-X_{M}\right)^{+}$.

A trading strategy is given by two stochastic processes $\left(\xi_{k}\right)_{k=0,1, \ldots, M}$ and $\left(\eta_{k}\right)_{k=0,1, \ldots, M}$, where $\xi_{k}$ is the number of shares held at time $t_{k}$ and $\eta_{k}$ is the amount invested in the bond at time $t_{k}$. We assume $\xi_{k}, \eta_{k}$ are $\mathcal{F}_{k}$-measurable, for all $0 \leq k \leq M$ and $\xi_{M}=0$. Consider the portfolio consisting of the combination of the stock and bond given by the trading strategy. The condition $\xi_{M}=0$ corresponds to the fact that at time $M$ we liquidate the portfolio in order to cover for the payoff of the option. The value of the portfolio at any time $t_{k}, \quad 0 \leq k \leq M$, is given by:

$$
V_{k}=\xi_{k} X_{k}+\eta_{k}
$$

For all $0 \leq j \leq M-1, \xi_{j}\left(X_{j+1}-X_{j}\right)$ represents the change in value due to the change in the stock price at time $t_{j+1}$ before any changes in the portfolio. Therefore, the accumulated gain $G_{k}$ is given by:

$$
G_{k}=\sum_{j=0}^{k-1} \xi_{j}\left(X_{j+1}-X_{j}\right), \quad 1 \leq k \leq M
$$

and $G_{0}=0$.

The cumulative cost at time $t_{k}, C_{k}$, is defined by:

$$
C_{k}=V_{k}-G_{k}, \quad 0 \leq k \leq M .
$$

A strategy is called self-financing if its cumulative cost process $\left(C_{k}\right)_{k=0,1, \ldots, M}$ is constant over time, i.e. $C_{0}=C_{1}=\ldots=C_{M}$. This is equivalent to $\left(\xi_{k+1}-\xi_{k}\right) X_{k+1}+\eta_{k+1}-\eta_{k}=0$ (a.s.), for all $0 \leq k \leq M-1$. In other words, any fluctuations in the stock price can be neutralized by rebalancing $\xi$ and $\eta$ with no inflow or outflow of capital. The value of the portfolio for a self-financing strategy is then given by $V_{k}=V_{0}+G_{k}$ at any time $0 \leq k \leq M$.

A market is complete if any claim $H$ is attainable, that is, there exists a self-financing strategy with $V_{M}=H$ (a.s.). If the market is incomplete, for instance in the case of discrete hedging, a claim is, in general, non-attainable and a hedging strategy has to be chosen based on some optimality criterion.

One approach to hedging in an incomplete market is to consider only self-financing strategies. An optimal self-financing strategy is then chosen which best approximates $H$ by 
its terminal value $V_{M}$. The quadratic criterion for this total risk-minimization is given by minimizing the $L^{2}$-norm:

$$
E\left(\left(H-V_{M}\right)^{2}\right)=E\left(\left(H-V_{0}-G_{M}\right)^{2}\right) .
$$

By solving the total risk-minimization problem (1), we obtain the initial value of the portfolio, $V_{0}$, and the number of shares, $\left(\xi_{0}, \ldots, \xi_{M-1}\right)$. The amount invested in the bond, $\left(\eta_{0}, \ldots, \eta_{M}\right)$, is then uniquely determined since the strategy is self-financing. Unfortunately, this problem does not have solution in general. Schweizer (1995) proves the existence of a total risk-minimizing strategy when the discounted stock price has a bounded mean-variance tradeoff, that is:

$$
\frac{\left(E\left(X_{k}-X_{k-1} \mid \mathcal{F}_{k}\right)\right)^{2}}{\operatorname{Var}\left(X_{k}-X_{k-1} \mid \mathcal{F}_{k}\right)} \text { is P-a.s. uniformly bounded. }
$$

Another approach to hedging in an incomplete market is to first impose $V_{M}=H$, hence $\eta_{M}=H$. Since such a strategy cannot be self-financing, we should then choose the optimal trading strategy to minimize the incremental cost incurred from adjusting the portfolio at each hedging time. The quadratic criterion for this local risk-minimizing strategy is given by minimizing:

$$
E\left(\left(C_{k+1}-C_{k}\right)^{2} \mid \mathcal{F}_{k}\right), 0 \leq k \leq M-1 .
$$

When $M=1$, the local risk-minimization and the total risk-minimization criteria coincide. Under the assumption that the above mean-variance tradeoff is deterministic, Schäl (1994) proves that the initial cost for the local risk-minimizing strategy is equal to the cost for the total risk-minimizing strategy. He then infers that this cost is a fair hedging price. However, this assumption is strong and in general the two initial costs are different.

These two criteria are discussed in detail in Föllmer, and Schweizer (1989), Schäl (1994), Schweizer $(1995,2001)$. We will concentrate here only on the local risk-minimization (2).

The problem is to minimize the local risk:

$$
E\left(\left(C_{k+1}-C_{k}\right)^{2} \mid \mathcal{F}_{k}\right)=E\left(\left(V_{k+1}-V_{k}-\xi_{k}\left(X_{k+1}-X_{k}\right)\right)^{2} \mid \mathcal{F}_{k}\right),
$$

for all $0 \leq k \leq M-1$, starting from the final condition $V_{M}=H$.

This local risk-minimization strategy has also a "global aspect": in order to determine the holdings in the hedging portfolio at a certain time, optimization problems need to be solved backward in time from the maturity of the option. Since the market is incomplete, the option values are not known at intermediate times and therefore, the hedging strategy cannot consider only the information in the current hedging period.

If $H$ is a square integrable random variable and $X$ is a square integrable process with bounded mean-variance tradeoff, then (2) is guaranteed to have a solution obtained in the following way: starting from $V_{M}=\eta_{M}=H$, for $k=M-1, \ldots, 0$ we choose $\xi_{k}, \eta_{k}$ recursively to minimize,

$$
E\left(\left(V_{k+1}-V_{k}-\xi_{k}\left(X_{k+1}-X_{k}\right)\right)^{2} \mid \mathcal{F}_{k}\right)=E\left(\left(X_{k+1}\left(\xi_{k+1}-\xi_{k}\right)+\left(\eta_{k+1}-\eta_{k}\right)\right)^{2} \mid \mathcal{F}_{k}\right) .
$$

The hedging strategy constructed in this way is given explicitly by:

$$
\begin{cases}\xi_{M}=0, \eta_{M}=H & \\ \xi_{k}=\frac{\operatorname{Cov}\left(\xi_{k+1} X_{k+1}+\eta_{k+1}, X_{k+1} \mid \mathcal{F}_{k}\right)}{\operatorname{Var}\left(X_{k+1} \mid \mathcal{F}_{k}\right)}, & 0 \leq k \leq M-1 \\ \eta_{k}=E\left(\left(\xi_{k+1}-\xi_{k}\right) X_{k+1}+\eta_{k+1} \mid \mathcal{F}_{k}\right), & 0 \leq k \leq M-1 .\end{cases}
$$


This hedging strategy is no longer self-financing, but it has a weaker property: it is mean-self-financing, that is $E\left(C_{k+1} \mid \mathcal{F}_{k}\right)=C_{k}$, for all $0 \leq k \leq M-1$, or, in other words, the cost process is a martingale. In particular, this implies $C_{0}=E\left(C_{M}\right)$. One could choose $C_{0}=V_{0}$ as a fair hedging price, however, $C_{0}$ depends on the subjective criterion for measuring the risk and it may not always make sense from an economic point of view (see Schweizer (1995)).

\section{Piecewise linear local risk-minimization}

An alternative way for choosing a local risk-minimizing strategy is to use the risk measure $E\left(\left|C_{k+1}-C_{k}\right| \mid \mathcal{F}_{k}\right)$ in the above minimization problem. The problem of choosing a piecewise linear local risk-minimizing strategy is then given by: starting from the final condition $V_{M}=H$, minimize,

$$
E\left(\left|C_{k+1}-C_{k}\right| \mid \mathcal{F}_{k}\right), 0 \leq k \leq M-1 .
$$

Problem (5) seems more natural than (2) from the financial point of view, since we are trying to minimize the risk measured by the incremental cost for adjusting the portfolio valued in monetary units. Moreover, as shown in the Appendix, when the risky asset price follows a binomial model, problems (2) and (5) respectively reduce to solving $L^{2}$ and $L^{1}$ minimization problems. The fact that the $L^{2}$-norm overemphasizes the large values even if these values have a very small probability of occurrence results in a poorer fit globally. This is another reason why we believe that problem (5) is more appropriate than (2). We believe the choice of the quadratic risk-minimization criterion in literature was made mainly because it allows explicit results, but we will see that using piecewise linear risk-minimization often leads to significantly different hedging strategies and possibly better hedging results.

Similar to the case of the quadratic criterion, the optimal piecewise linear strategy is constructed recursively by first choosing $\eta_{M}=H, \xi_{M}=0$, then for $k=M-1, \ldots, 0$ choosing $\xi_{k}, \eta_{k}$ to minimize:

$$
E\left(\left|X_{k+1}\left(\xi_{k+1}-\xi_{k}\right)+\left(\eta_{k+1}-\eta_{k}\right)\right| \mid \mathcal{F}_{k}\right) .
$$

The strategy constructed in this way is no longer mean-self-financing. The lack of this property is not a practical drawback since we are predominantly interested in reducing the mean of the incremental costs and not in preserving the mean cost. However, if we insist on mean-self-financing strategies, we can solve instead the following local risk-minimization problem: starting from $V_{M}=H$, for all $k=M-1, \ldots, 0$, minimize

$$
\left\{\begin{array}{l}
\min E\left(\left|C_{k+1}-C_{k}\right| \mid \mathcal{F}_{k}\right) \\
\text { subject to } E\left(C_{k+1} \mid \mathcal{F}_{k}\right)=C_{k} .
\end{array}\right.
$$

That is, first take $\eta_{M}=H, \xi_{M}=0$, then recursively, for $k=M-1, \ldots, 0$, choose $\xi_{k}$ to minimize:

$$
E\left(\left|\xi_{k+1} X_{k+1}+\eta_{k+1}-E\left(\xi_{k+1} X_{k+1}+\eta_{k+1} \mid \mathcal{F}_{k}\right)-\xi_{k}\left(X_{k+1}-E\left(X_{k+1} \mid \mathcal{F}_{k}\right)\right)\right| \mid \mathcal{F}_{k}\right)
$$

and define $\eta_{k}=E\left(\xi_{k+1} X_{k+1}+\eta_{k+1}-\xi_{k} X_{k+1} \mid \mathcal{F}_{k}\right)$.

We note that while in problem (6) we have the liberty of choosing both $\eta_{k}$ and $\xi_{k}$, the mean-self-financing constraint imposes a relation between these two variables.

Unfortunately, it is not possible to obtain an analytic solution for problems (6) and (8). 


\section{Numerical results}

Assume the writer of a European option with maturity $T$ wants to hedge his position using the underlying stock and a bond. Also assume there are only $M$ hedging opportunities at $0=t_{0}<t_{1}<\ldots<t_{M-1}<t_{M}:=T$.

Suppose the price of the underlying stock follows the stochastic differential equation:

$$
\frac{d S_{t}}{S_{t}}=\mu d t+\sigma d Z_{t}
$$

where $Z_{t}$ is a Wiener process.

We generate a binomial tree of possible values of the asset price choosing the parameters such that if the number of periods in the tree is increased, in the limiting case, the binomial process converges to the continuous process (9). The numerical results presented in this section refer to hedging put options with maturity $T=1$ and different strike prices. We have performed numerical experiments for different values of the instantaneous expected return, $\mu$, volatility, $\sigma$, and riskless rate of return, $r$. Unless otherwise specified, the results presented here have been obtained for the initial value of the stock $S_{0}=100, \mu=.2, \sigma=.2$ and $r=.1$. The number of periods in the binomial tree is 600 . The detailed implementation of all the three methods when using a binomial tree is presented in the Appendix.

We compute the holdings $(\xi, \eta)$ in the portfolio at each node in the binomial tree using the previously described methods of local risk-minimization:

- Method 1: Piecewise linear risk-minimization (6)

- Method 2: Quadratic risk-minimization (4)

- Method 3: Constrained piecewise linear risk-minimization (8)

We want to analyze the performance of the three methods given that the stock price satisfies equation (9). We generate synthetic paths for the stock price based on this equation. For each path and each of the three methods we determine the holdings in the portfolio used to hedge the option if the stock price had the values given by the path. The portfolio holdings are determined based on the already computed holdings in the binomial tree: at every hedging time we find the node in the binomial tree where the stock price has the closest value to the one on the path and we consider the holdings at that particular node. We then compute the following:

- End of period cumulative cost:

$$
C_{M}=H-\sum_{k=0}^{M-1} \xi_{k}\left(X_{k+1}-X_{k}\right) .
$$

This is the total amount of money necessary for the writer to carry on the hedging strategy and honor the option payoff.

- Incremental risk per rebalancing time:

$$
\frac{1}{M} \sum_{k=0}^{M-1}\left|C_{k+1}-C_{k}\right| .
$$


This is the average of the absolute values of all the adjustments in the portfolio. It is a measure of the unplanned intermediate costs or income of the strategy. It is not a priori clear that the incremental risk for Method 1 should be smaller than the incremental risk for the other two methods: Method 1 minimizes $E\left(\left|C_{k+1}-C_{k}\right| \mid \mathcal{F}_{k}\right)$, for $k=M-1, \ldots, 0$, and not their sum. As shown below, it actually happens that Method 1 yields a larger incremental risk than the other two methods.

The numerical results will show that the performance of the three methods depends on the moneyness of the options. Method 1, the piecewise linear risk minimization, yields the smallest average cumulative cost and incremental risk among the three methods when the put options are out-of-money and at-the-money, while Method 2, the quadratic risk minimization, performs the best as the put options become more deeply in-the-money. Method 3, the constrained piecewise linear risk minimization, is an intermediate method. The cumulative cost and incremental risk for Method 3 follow the trend of the cumulative cost and incremental risk for Method 1, but their values are closer to the values for Method 2. The numerical results will also illustrate that the differences between the three methods tend to increase as we rebalance less frequently.

Let us first analyze the case of the static hedging when we only hedge once, at time 0 . Tables 1 and 2 show the average cumulative cost and incremental risk over 50000 simulated paths. 
Table 1: Average cumulative cost over 50000 paths for one hedging opportunity

\begin{tabular}{|c|c|c|c|}
\cline { 2 - 4 } \multicolumn{1}{c|}{} & \multicolumn{3}{c|}{ Method } \\
\hline Strike & 1 & 2 & 3 \\
\hline 90 & 0.5194 & 0.9598 & 0.6481 \\
\hline 95 & 0.9672 & 1.7250 & 1.2565 \\
\hline 100 & 1.6619 & 2.8605 & 2.2338 \\
\hline 105 & 2.6633 & 4.4282 & 3.7382 \\
\hline 110 & 4.9958 & 6.4504 & 5.9590 \\
\hline
\end{tabular}

The table shows the average cumulative cost for the static hedging of put options with maturity $T=1$ and different strike prices. The hedging strategies are computed by the three methods: 1 - piecewise linear riskminimization, 2 - quadratic risk-minimization and 3 - constrained piecewise linear risk-minimization. The results are obtained for the initial value of the stock, $S_{0}=100$, instantaneous expected return, $\mu=.2$, volatility, $\sigma=.2$, and riskless rate of return, $r=.1$.

Table 2: Average incremental risk over 50000 paths for one hedging opportunity

\begin{tabular}{|c|c|c|c|}
\cline { 2 - 4 } \multicolumn{1}{c|}{} & \multicolumn{3}{c|}{ Method } \\
\hline Strike & 1 & 2 & 3 \\
\hline 90 & 0.5194 & 1.1294 & 0.9156 \\
\hline 95 & 0.9672 & 1.8111 & 1.5629 \\
\hline 100 & 1.6619 & 2.6146 & 2.3863 \\
\hline 105 & 2.6633 & 3.4483 & 3.2889 \\
\hline 110 & 3.8395 & 4.2187 & 4.1471 \\
\hline
\end{tabular}

The table shows the average incremental risk for the static hedging of put options with different strike prices, by the three methods and in the setup described in Table 1.

The average cumulative cost for Methods 1 and 3 is much smaller than the cost for Method 2. This is especially the case for Method 1 for which the cumulative cost is almost two thirds the cost of Method 2.

Similar results are illustrated in Table 2. Since the incremental risk accumulates the absolute values of all the unplanned costs or profits of the strategy, it is a measure of the riskiness of the strategy. Using this risk measure, we infer that Method 1 is the least risky among the three methods. Method 3 is also less risky than Method 2.

We remark that, in the case of Method 1, the average values of the cumulative cost in Table 1 and incremental risk in Table 2 are equal for the first four put options considered. This happens because both $\xi_{0}$ and $\eta_{0}$ are zero. Therefore, with no future rebalancing opportunities, Method 1 chooses not to hedge these particular put options. However, the more deeply in-the-money put option with strike price 110 is hedged by Method 1, that is, the holdings in the hedging portfolio are no longer zero. Experiments show that in-themoney put options which are closer to expiry will also be hedged by Method 1 .

Consider now that we have more hedging opportunities. Tables 3 and 4 show the average 
values over 50000 paths of the cumulative cost and incremental risk, for different number of binomial tree periods per rebalancing time. We remark that the last column corresponds to the above case, that is, hedging only once, at time 0.

Table 3: Average value of the cumulative cost over 50000 paths

\begin{tabular}{|c|c|c|c|c|c|c|c|c|}
\cline { 3 - 9 } \multicolumn{1}{c|}{} & \multicolumn{7}{c|}{ Number of periods per rebalancing time } \\
\hline Strike & Method & 1 & 5 & 25 & 50 & 100 & 300 & 600 \\
\hline \hline \multirow{3}{*}{90} & 1 & 1.4257 & 0.9297 & 1.1807 & 0.9127 & 0.8219 & 0.6253 & 0.5194 \\
\cline { 2 - 9 } & 2 & 1.4257 & 1.4214 & 1.3992 & 1.3707 & 1.3148 & 1.1338 & 0.9598 \\
\cline { 2 - 9 } & 3 & 1.4257 & 1.3729 & 1.3085 & 1.2677 & 1.2071 & 0.8728 & 0.6481 \\
\hline \hline \multirow{3}{*}{95} & 1 & 2.3993 & 1.7981 & 2.1335 & 1.7798 & 1.6810 & 1.1994 & 0.9672 \\
\cline { 2 - 9 } & 2 & 2.3993 & 2.3926 & 2.3633 & 2.3212 & 2.2480 & 1.9919 & 1.7250 \\
\cline { 2 - 9 } & 3 & 2.3993 & 2.3300 & 2.2501 & 2.1984 & 2.1412 & 1.6418 & 1.2565 \\
\hline \hline \multirow{3}{*}{100} & 1 & 3.7547 & 3.1753 & 3.5134 & 3.1471 & 2.9982 & 2.1141 & 1.6619 \\
\cline { 2 - 9 } & 2 & 3.7547 & 3.7459 & 3.7124 & 3.6666 & 3.5681 & 3.2349 & 2.8605 \\
\cline { 2 - 9 } & 3 & 3.7547 & 3.6715 & 3.5838 & 3.5367 & 3.4839 & 2.8866 & 2.2338 \\
\hline \hline \multirow{3}{*}{105} & 1 & 5.5251 & 5.1157 & 5.3573 & 5.0783 & 4.9656 & 4.3046 & 2.6633 \\
\cline { 2 - 9 } & 2 & 5.5251 & 5.5179 & 5.4826 & 5.4299 & 5.3158 & 4.9137 & 4.4282 \\
\cline { 2 - 9 } & 3 & 5.5251 & 5.4359 & 5.3481 & 5.3036 & 5.2763 & 4.6740 & 3.7382 \\
\hline \hline & 1 & 7.7189 & 7.6090 & 7.6409 & 7.5231 & 7.4193 & 6.9338 & 4.9958 \\
\cline { 2 - 9 } & 2 & 7.7189 & 7.7120 & 7.6701 & 7.6148 & 7.4915 & 7.0380 & 6.4504 \\
\cline { 2 - 9 } & 3 & 7.7189 & 7.6264 & 7.5365 & 7.5048 & 7.5083 & 7.0088 & 5.9590 \\
\hline
\end{tabular}

The table shows the average cumulative cost for the hedging of put options with different strike prices and number of periods per rebalancing time, by the three methods and in the setup described in Table 1. 
Table 4: Average value of the incremental risk over 50000 paths

\begin{tabular}{|c|c|c|c|c|c|c|c|c|}
\cline { 3 - 9 } \multicolumn{1}{c|}{} & \multicolumn{7}{c|}{ Number of periods per rebalancing time } \\
\hline Strike & Method & 1 & 5 & 25 & 50 & 100 & 300 & 600 \\
\hline \hline \multirow{3}{*}{90} & 1 & 0.0035 & 0.0088 & 0.0557 & 0.0874 & 0.1686 & 0.3676 & 0.5194 \\
\cline { 2 - 9 } & 2 & 0.0035 & 0.0142 & 0.0667 & 0.1319 & 0.2580 & 0.6903 & 1.1294 \\
\cline { 2 - 9 } & 3 & 0.0035 & 0.0147 & 0.0672 & 0.1304 & 0.2520 & 0.6160 & 0.9156 \\
\hline \hline \multirow{3}{*}{95} & 1 & 0.0049 & 0.0160 & 0.0843 & 0.1545 & 0.3006 & 0.7081 & 0.9672 \\
\cline { 2 - 9 } & 2 & 0.0049 & 0.0197 & 0.0935 & 0.1861 & 0.3696 & 1.0397 & 1.8111 \\
\cline { 2 - 9 } & 3 & 0.0049 & 0.0206 & 0.0951 & 0.1860 & 0.3657 & 0.9845 & 1.5629 \\
\hline \hline \multirow{3}{*}{100} & 1 & 0.0063 & 0.0256 & 0.1141 & 0.2362 & 0.4624 & 1.2406 & 1.6619 \\
\cline { 2 - 9 } & 2 & 0.0063 & 0.0253 & 0.1205 & 0.2418 & 0.4849 & 1.4254 & 2.6146 \\
\cline { 2 - 9 } & 3 & 0.0063 & 0.0265 & 0.1233 & 0.2430 & 0.4821 & 1.4100 & 2.3863 \\
\hline \hline \multirow{3}{*}{1105} & 1 & 0.0075 & 0.0350 & 0.1414 & 0.3101 & 0.6074 & 1.8267 & 2.6633 \\
\cline { 2 - 9 } & 2 & 0.0075 & 0.0302 & 0.1443 & 0.2911 & 0.5900 & 1.7990 & 3.4483 \\
\cline { 2 - 9 } & 3 & 0.0075 & 0.0316 & 0.1481 & 0.2930 & 0.5857 & 1.8274 & 3.2889 \\
\cline { 2 - 9 } & 2 & 0.0085 & 0.0418 & 0.1603 & 0.3622 & 0.7128 & 2.1912 & 3.8395 \\
\cline { 2 - 9 } & 3 & 0.0085 & 0.0338 & 0.1623 & 0.3286 & 0.6725 & 2.1217 & 4.2187 \\
\hline \hline
\end{tabular}

The table shows the average incremental risk for the hedging of put options with different strike prices and number of periods per rebalancing time, by the three methods and in the setup described in Table 1.

Consistent with the single hedging opportunity case, the average cumulative cost for Method 1 is the smallest. Method 3 has an average cumulative cost close in value to Method 2, although smaller in most of the cases. As mentioned above, the differences tend to increase when rebalancing is infrequent. With respect to the risk measure chosen, Method 1 is the best for the out-of-money and at-the-money put. The incremental risk for Method 3 is also smaller (or very close) to the risk for Method 2. In the case of the in-the-money put, Method 2 tends to be marginally better if we rebalance frequently enough.

The fact that Method 2 performs better than the other two methods as the put options become more deeply in-the-money and the rebalancing is frequent enough is illustrated by considering put options that are more likely to mature in the money. Suppose now that the instantaneous expected return is $\mu=.15$ and the riskless rate of return is $r=.04$ while the other parameters remain unchanged. The average cumulative cost over 50000 for the put options in this case is presented in Table 5. 
Table 5: Average value of the cumulative cost over 50000 paths

\begin{tabular}{|c|c|c|c|c|c|c|c|c|}
\cline { 3 - 9 } \multicolumn{2}{c|}{} & \multicolumn{7}{c|}{ Number of periods per rebalancing time } \\
\hline Strike & Method & 1 & 5 & 25 & 50 & 100 & 300 & 600 \\
\hline \hline \multirow{3}{*}{90} & 1 & 2.5329 & 1.8672 & 2.2014 & 2.1110 & 1.8588 & 1.1700 & 0.9339 \\
\cline { 2 - 9 } & 2 & 2.5329 & 2.5266 & 2.4908 & 2.4449 & 2.3506 & 2.0470 & 1.7414 \\
\cline { 2 - 9 } & 3 & 2.5329 & 2.4521 & 2.5587 & 2.4527 & 2.1765 & 1.6673 & 1.2394 \\
\hline \hline \multirow{3}{*}{95} & 1 & 4.0342 & 3.3979 & 3.7302 & 3.6446 & 3.3893 & 2.1354 & 1.6568 \\
\cline { 2 - 9 } & 2 & 4.0342 & 4.0311 & 3.9842 & 3.9188 & 3.8077 & 3.4075 & 2.9697 \\
\cline { 2 - 9 } & 3 & 4.0342 & 3.9429 & 4.0780 & 3.9603 & 3.6461 & 3.0212 & 2.2798 \\
\hline \hline \multirow{3}{*}{100} & 1 & 6.0060 & 5.5982 & 5.7886 & 5.7813 & 5.5293 & 4.3262 & 2.7203 \\
\cline { 2 - 9 } & 2 & 6.0060 & 5.9979 & 5.9554 & 5.8905 & 5.7519 & 5.2661 & 4.6908 \\
\cline { 2 - 9 } & 3 & 6.0060 & 5.8998 & 6.0735 & 5.9669 & 5.6390 & 4.9550 & 3.9174 \\
\hline \hline \multirow{3}{*}{105} & 1 & 8.4531 & 8.4119 & 8.3506 & 8.4183 & 8.1996 & 7.4385 & 5.4375 \\
\cline { 2 - 9 } & 2 & 8.4531 & 8.4454 & 8.3962 & 8.3285 & 8.1803 & 7.6329 & 6.9312 \\
\cline { 2 - 9 } & 3 & 8.4531 & 8.3455 & 8.5315 & 8.4374 & 8.1286 & 7.4884 & 6.3763 \\
\hline \hline & 1 & 11.3490 & 11.6763 & 11.3757 & 11.5254 & 11.3399 & 11.0763 & 9.2010 \\
\cline { 2 - 8 } & 2 & 11.3490 & 11.3409 & 11.2905 & 11.2235 & 11.0757 & 10.4973 & 9.6961 \\
\cline { 2 - 8 } & 3 & 11.3490 & 11.2431 & 11.4351 & 11.3555 & 11.0830 & 10.7201 & 9.5060 \\
\hline
\end{tabular}

The table shows the average cumulative cost for the hedging of put options with different strike prices and number of periods per rebalancing time, by the three methods described in Table 1 . The results are obtained for the instantaneous expected return, $\mu=.15$ and the riskless rate of return, $r=.04$, the other parameters being unchanged.

We remark that for the out-of-money and at-the-money put options, Method 1 has the smallest average cumulative cost. As the option is more deeply in-the-money, Method 2 tends to perform the best in terms of average cumulative cost for frequent enough rebalancing. However, the relative differences between the average cumulative costs for Methods 1 and 2 are much smaller for the in-the-money put options than for the out-of-money put options. Method 3 has an average cumulative cost close in value to the cost of Method 2, although smaller when rebalancing is infrequent and the option is not deep in-the-money.

The values of the incremental risk for these put options are very close and we are not going to present them. However, they follow the same trend: Method 1 has the smallest incremental risk for out-of-money and at-the-money put options, while Method 2 slightly outperforms it when the options are in-the-money.

The expected total cost and incremental risk can be approximated even without simulation by analyzing the three methods in the binomial view of the world, where the stock price can only follow the paths given by the binomial tree. For each method we compute the expected cumulative cost and expected incremental risk over the entire binomial tree.

- Expected time T cumulative cost:

$$
E\left(C_{M}\right)=E\left(H-\sum_{k=0}^{M-1} \xi_{k}\left(X_{k+1}-X_{k}\right)\right) .
$$

This is the expected cost of the hedging strategy which can be easily computed once 
$\xi_{k}$ has been computed. It is an indication of how much the hedging strategy costs on average.

- Expected incremental risk per rebalancing time:

$$
E\left(\frac{1}{M} \sum_{k=0}^{M-1} E\left(\left|C_{k+1}-C_{k}\right| \mid \mathcal{F}_{k}\right)\right)=\frac{1}{M} \sum_{k=0}^{M-1} E\left(\left|C_{k+1}-C_{k}\right|\right)
$$

This is a measure of the average unplanned intermediate costs or income of the strategy. As mentioned before, it is not a priori clear that the expected incremental risk for Method 1 should be the smallest.

The results that follow have been obtained with the first set of parameters where the instantaneous expected return is $\mu=.15$ and the riskless rate of return is $r=.04$.

The expected cost for all three methods is presented in Table 6.

Table 6: Expected cumulative cost $E\left(C_{M}\right)$

\begin{tabular}{|c|c|c|c|c|c|c|c|c|}
\cline { 3 - 9 } \multicolumn{1}{c|}{} & \multicolumn{8}{c|}{ Number of periods per rebalancing time } \\
\hline Strike & Method & 1 & 5 & 25 & 50 & 100 & 300 & 600 \\
\hline \hline \multirow{3}{*}{90} & 1 & 1.4254 & 0.9289 & 1.1780 & 0.9095 & 0.8170 & 0.6228 & 0.5213 \\
\cline { 2 - 10 } & 2 & 1.4254 & 1.4204 & 1.3962 & 1.3669 & 1.3118 & 1.1348 & 0.9671 \\
\cline { 2 - 10 } & 3 & 1.4254 & 1.3718 & 1.3057 & 1.2640 & 1.2030 & 0.8722 & 0.6516 \\
\hline \hline \multirow{3}{*}{95} & 1 & 2.3977 & 1.7969 & 2.1282 & 1.7763 & 1.6797 & 1.1971 & 0.9682 \\
\cline { 2 - 10 } & 2 & 2.3977 & 2.3912 & 2.3593 & 2.3203 & 2.2455 & 1.9929 & 1.7353 \\
\cline { 2 - 10 } & 3 & 2.3977 & 2.3284 & 2.2460 & 2.1976 & 2.1381 & 1.6401 & 1.2611 \\
\hline \hline \multirow{3}{*}{100} & 1 & 3.7499 & 3.1634 & 3.5010 & 3.1313 & 2.9887 & 2.1038 & 1.6570 \\
\cline { 2 - 9 } & 2 & 3.7499 & 3.7422 & 3.7035 & 3.6557 & 3.5626 & 3.2321 & 2.8703 \\
\cline { 2 - 9 } & 3 & 3.7499 & 3.6674 & 3.5739 & 3.5236 & 3.4766 & 2.8802 & 2.2359 \\
\hline \hline \multirow{3}{*}{1105} & 1 & 5.5191 & 5.1031 & 5.3356 & 5.0523 & 4.9430 & 4.2796 & 2.6471 \\
\cline { 2 - 9 } & 2 & 5.5191 & 5.5103 & 5.4667 & 5.4122 & 5.3045 & 4.9042 & 4.4337 \\
\cline { 2 - 9 } & 3 & 5.5191 & 5.4274 & 5.3294 & 5.2836 & 5.2607 & 4.6578 & 3.7352 \\
\cline { 2 - 9 } & 2 & 7.7139 & 7.5957 & 7.6202 & 7.4950 & 7.3966 & 6.9102 & 4.9857 \\
\hline \hline & 3 & 7.7139 & 7.7046 & 7.6583 & 7.6000 & 7.4833 & 7.0297 & 6.4581 \\
\hline \hline
\end{tabular}

The table shows the expected cumulative cost for the hedging of put options with different strike prices and number of periods per rebalancing time, by the three methods and in the setup described in Table 1.

We observe that when we rebalance the portfolio every period, all the methods yield the same expected cost since we are able to exactly replicate the options on all the paths of the binomial tree. When the number of periods per rebalancing time is larger than 1 , Method 1 gives a smaller expected cost than Method 2. Method 3 is also better than Method 2 but the differences are less significant. We remark also that the expected cost for piecewise linear local risk-minimization is much smaller when we rebalance infrequently. 
Let us analyze now the expected incremental risk.

Table 7: Expected incremental risk per rebalancing time

\begin{tabular}{|c|c|c|c|c|c|c|c|c|}
\cline { 3 - 9 } \multicolumn{1}{c|}{} & \multicolumn{8}{c|}{ Number of periods per rebalancing time } \\
\hline Strike & Method & 1 & 5 & 25 & 50 & 100 & 300 & 600 \\
\hline \hline \multirow{3}{*}{90} & 1 & 0.0000 & 0.0075 & 0.0531 & 0.0858 & 0.1672 & 0.3637 & 0.5213 \\
\cline { 2 - 10 } & 2 & 0.0000 & 0.0135 & 0.0656 & 0.1302 & 0.2562 & 0.6842 & 1.1273 \\
\cline { 2 - 9 } & 3 & 0.0000 & 0.0129 & 0.0651 & 0.1283 & 0.2496 & 0.6111 & 0.9133 \\
\hline \hline \multirow{3}{*}{95} & 1 & 0.0000 & 0.0137 & 0.0800 & 0.1515 & 0.2982 & 0.7046 & 0.9682 \\
\cline { 2 - 10 } & 2 & 0.0000 & 0.0188 & 0.0921 & 0.1841 & 0.3672 & 1.0339 & 1.8108 \\
\cline { 2 - 9 } & 3 & 0.0000 & 0.0180 & 0.0919 & 0.1829 & 0.3622 & 0.9792 & 1.5635 \\
\hline \hline \multirow{3}{*}{100} & 1 & 0.0000 & 0.0217 & 0.1076 & 0.2296 & 0.4555 & 1.2355 & 1.6570 \\
\cline { 2 - 9 } & 2 & 0.0000 & 0.0241 & 0.1188 & 0.2389 & 0.4817 & 1.4197 & 2.6152 \\
\cline { 2 - 9 } & 3 & 0.0000 & 0.0231 & 0.1189 & 0.2381 & 0.4775 & 1.4054 & 2.3824 \\
\hline \hline \multirow{3}{*}{1105} & 1 & 0.0000 & 0.0299 & 0.1332 & 0.3014 & 0.5979 & 1.8135 & 2.6471 \\
\cline { 2 - 9 } & 2 & 0.0000 & 0.0287 & 0.1423 & 0.2878 & 0.5856 & 1.7967 & 3.4558 \\
\cline { 2 - 9 } & 3 & 0.0000 & 0.0275 & 0.1426 & 0.2867 & 0.5793 & 1.8207 & 3.2905 \\
\cline { 2 - 9 } & 2 & 0.0000 & 0.0357 & 0.1519 & 0.3530 & 0.7008 & 2.1749 & 3.8174 \\
\cline { 2 - 9 } & 3 & 0.0000 & 0.0321 & 0.1598 & 0.3246 & 0.6667 & 2.1156 & 4.2214 \\
\hline \hline
\end{tabular}

The table shows the expected incremental risk for the hedging of put options with different strike prices and number of periods per rebalancing time, by the three methods and in the setup described in Table 1.

When we rebalance the portfolio every period, we replicate the options exactly on all the paths of the binomial tree, therefore the risk is zero for all the methods. When we rebalance the portfolio less frequently, Method 1 yields the smallest expected incremental risk in most of the cases and Method 3 is an intermediate method. We notice again that the performance of the piecewise linear local risk-minimization depends on the moneyness of the put options, the best results being obtained for out-of-money and at-the-money put options. As shown in Table 7, the expected incremental risk per rebalancing time increases as we rebalance less frequently.

As we can see from Tables 6 and 7, the values of the expected total cost and expected incremental risk follow closely the values of the average cumulative cost and average incremental risk.

The above numerical results show that the three methods perform differently with respect to the total cost and incremental risk. Next we illustrate that their hedging styles are also different. Consider the particular case of hedging the out-of-money put option with strike price 95 and 12 hedging opportunities. We can compute the cumulative cost $C_{k}$ of the portfolio at each hedging time $t_{k}$ along a simulated path. Figure 2 shows the typical evolution of the cumulative cost along such a path. 


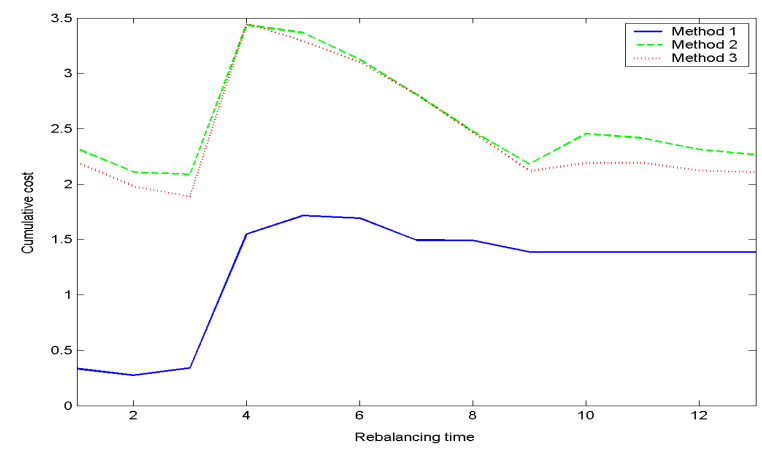

Figure 2: Cumulative costs along a simulated path for the stock price

We notice that the plots of the cumulative costs for the mean-self-financing Methods 2 and 3 are almost identical. Method 1 starts with a much smaller initial investment in the hedging portfolio and gradually increases it along the path in order to match the option payoff. The fact that the initial cost for setting the portfolio tends to be much smaller for Method 1 than for the other two methods is illustrated in Table 8. The initial portfolio cost is computed by $C_{0}=\xi_{0} X_{0}+\eta_{0}$, where $\xi_{0}, \eta_{0}$ are the holdings in the binomial tree at time 0 .

Table 8: Initial cost of the hedging portfolio

\begin{tabular}{|c|c|c|c|c|c|c|c|c|}
\cline { 3 - 9 } \multicolumn{2}{c|}{} & \multicolumn{6}{c|}{ Number of periods per rebalancing time } \\
\hline Strike & Method & 1 & 5 & 25 & 50 & 100 & 300 & 600 \\
\hline \hline \multirow{3}{*}{90} & 1 & 1.4254 & 0.0299 & 0.6442 & 0.0837 & 0.0000 & 0.0000 & 0.0000 \\
\cline { 2 - 9 } & 2 & 1.4254 & 1.4204 & 1.3962 & 1.3669 & 1.3188 & 1.1348 & 0.9671 \\
\cline { 2 - 9 } & 3 & 1.4254 & 1.3714 & 1.3057 & 1.2640 & 1.2030 & 0.8722 & 0.6516 \\
\hline \hline \multirow{3}{*}{95} & 1 & 2.3977 & 0.1530 & 1.3139 & 0.3328 & 0.3679 & 0.0000 & 0.0000 \\
\cline { 2 - 9 } & 2 & 2.3977 & 2.3912 & 2.3593 & 2.3203 & 2.2455 & 1.9929 & 1.7353 \\
\cline { 2 - 9 } & 3 & 2.3977 & 2.3284 & 2.2460 & 2.1976 & 2.1381 & 1.6401 & 1.2611 \\
\hline \hline \multirow{3}{*}{100} & 1 & 3.7499 & 0.5544 & 2.3361 & 0.8783 & 0.7925 & 0.0000 & 0.0000 \\
\cline { 2 - 9 } & 2 & 3.7499 & 3.7422 & 3.7035 & 3.6557 & 3.5626 & 3.2321 & 2.8703 \\
\cline { 2 - 9 } & 3 & 3.7499 & 3.6674 & 3.5739 & 3.5236 & 3.4766 & 2.8802 & 2.2359 \\
\hline \hline \multirow{3}{*}{110} & 1 & 5.5191 & 1.5201 & 4.0033 & 2.2123 & 2.4485 & 2.6349 & 0.0000 \\
\cline { 2 - 9 } & 2 & 5.5191 & 5.5103 & 5.4667 & 5.4122 & 5.3045 & 4.9042 & 4.4337 \\
\cline { 2 - 9 } & 3 & 5.5191 & 5.4274 & 5.3294 & 5.2836 & 5.2607 & 4.6578 & 3.7352 \\
\cline { 2 - 9 } & 2 & 7.7139 & 3.3123 & 6.1464 & 4.2234 & 4.1045 & 5.2699 & 2.6164 \\
\cline { 2 - 9 } & 3 & 7.7139 & 7.7046 & 7.6583 & 7.6000 & 7.4833 & 7.0297 & 6.4581 \\
\hline \hline
\end{tabular}

The table shows the initial cost of the hedging portfolio for put options with different strike prices and number of periods per rebalancing time, by the three methods and in the setup described in Table 1. 
From the last column in Table 8 we can see that when we only have one hedging opportunity, Method 1 yields $C_{0}=0$ for the first four put options considered. Actually, as emphasized before in the paper, both $\xi_{0}$ and $\eta_{0}$ are 0 . This is no longer the case if we hedge the more deeply in-the-money put option with strike price 105 and the same maturity, or in-the-money put options which are closer to expiry.

Next we analyze the distributions of the cumulative cost for the three methods with respect to the hedging of the out-of-money put option with 12 rebalancing opportunities. From Table 3 we see that the average cumulative costs for the three methods are 1.7798, 2.3212 and 2.1984, respectively. Figure 3 shows the histograms for the cumulative costs over the 50000 simulated paths.
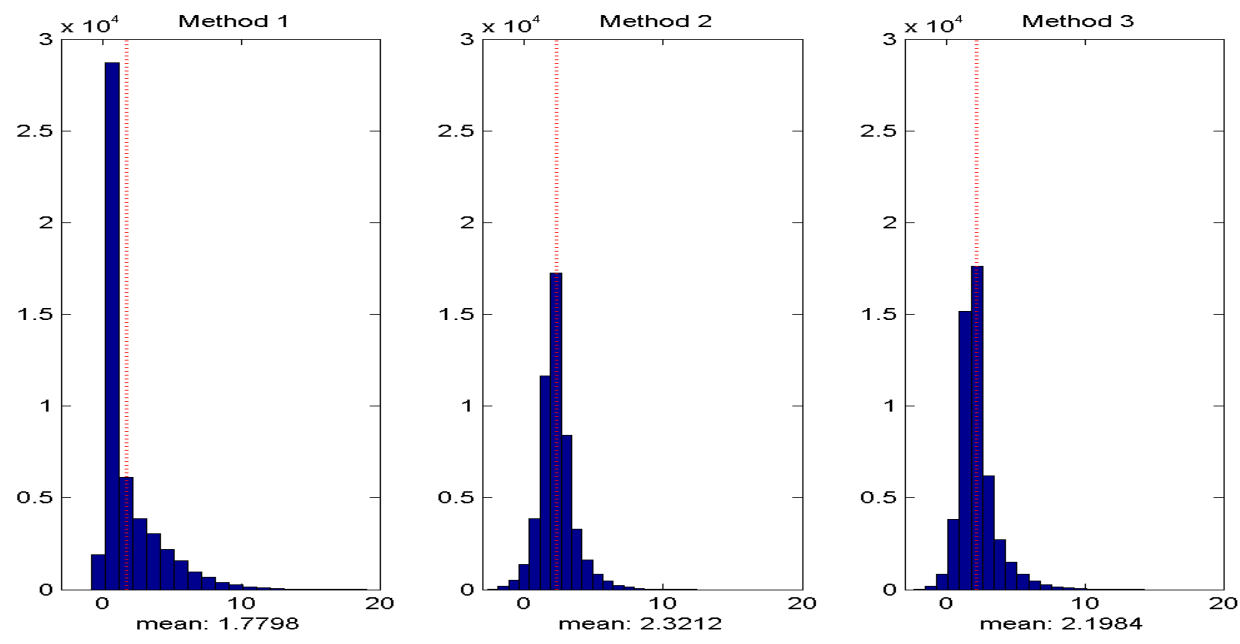

Figure 3: Histogram of cumulative costs

We can see that Method 1 is more asymmetric about its mean compared to Methods 2 and 3. Almost $70 \%$ of the time the cumulative costs for Method 1 are less than their mean and $53 \%$ are less than half of the mean. Since the average cumulative cost is very close to the expected total cost, it means that almost $70 \%$ of the time the cost for rebalancing the portfolio will be less than what we expect. In the case of Method 3,61\% of the time the cumulative costs are less than their mean, while in the case of Method 2 this happens only $56 \%$ of the time. However, Figure 3 also shows that Method 1 has a very small probability of having larger costs than the costs for the other two methods.

All the three methods can lead to negative cumulative costs, but this happens less frequently in the case of Method 1. Moreover, as we can see from Figure 3, the negative cumulative costs for Method 1 are closer in value to zero than the negative cumulative costs for Methods 2 and 3. The minimum cumulative cost over the 50000 simulated paths is -0.7598 for Method 1, -2.5966 for Method 2 and -2.3504 for Method 3 .

The skewness of the cumulative costs is another indication of the asymmetry of the data around the mean. In our case, the skewness for Method 1 is 2.1422, while the skewness for Method 2 is 1.0003 and the one for Method 3 is 1.7019. The larger positive skewness for Method 1 emphasizes again that the distribution of the cumulative costs for Method 1 has a longer right tail than the distributions for the other two methods.

The histograms for the incremental risks over the same paths are presented in Figure 4. The average incremental risks for the three methods are very close in value. However, the median for Method 1 is 0.0598 , while the medians for the other two methods are 0.1414 for 
Method 2 and 0.1330 for Method 3. On the other hand, as in the case of the cumulative cost, we can also notice that the incremental risk for Method 1 is more widely spread. The skewness for the three methods are $1.8667,1.3447$, and 1.4572 respectively.
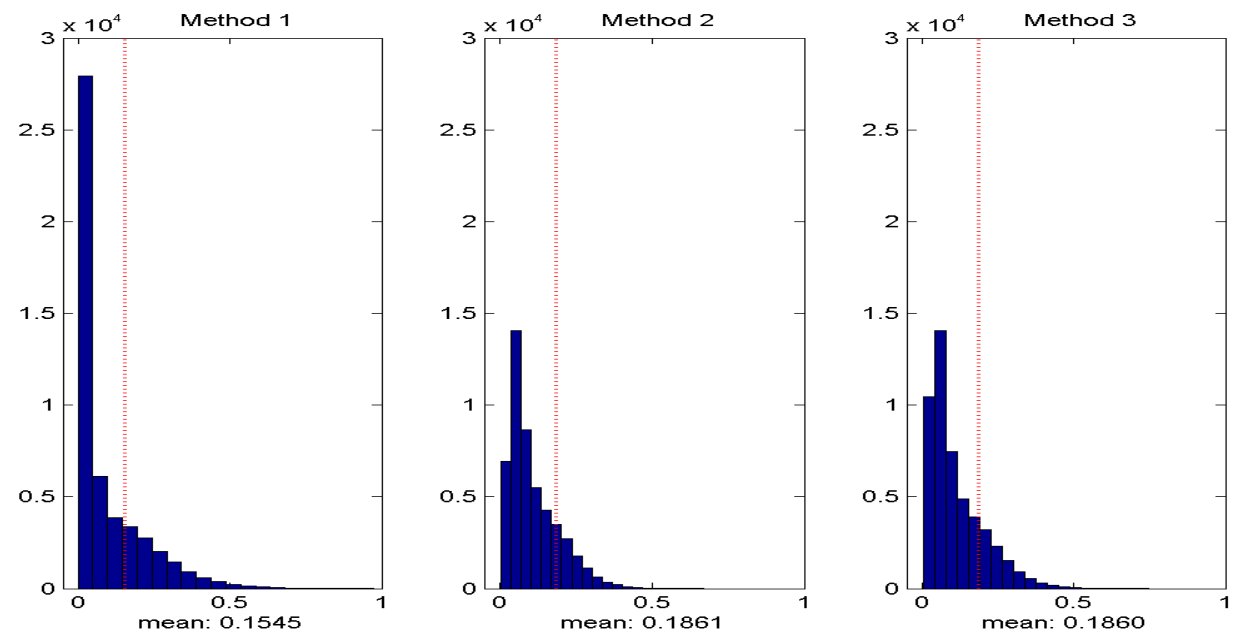

Figure 4: Histogram of incremental risks

The distributions of the cumulative cost and incremental risk become more and more asymmetrical as we hedge less frequently or the put option is more deeply out-of-money. Tables 9 and 10 show the skewness of the distributions of the cumulative cost and incremental risk for at-the-money and out-of-money puts with very few hedging opportunities.

Table 9: Skewness of the cumulative cost

\begin{tabular}{|c|c|c|c|c|}
\cline { 3 - 5 } \multicolumn{2}{c|}{} & \multicolumn{3}{c|}{$\begin{array}{c}\text { Number of periods per } \\
\text { rebalancing time }\end{array}$} \\
\hline \hline \multirow{3}{*}{ Strike } & Method & 100 & 300 & 600 \\
\hline \hline \multirow{3}{*}{95} & 1 & 1.9793 & 2.6503 & 3.4271 \\
\cline { 2 - 5 } & 2 & 1.0388 & 1.7781 & 2.4873 \\
\cline { 2 - 5 } & 3 & 1.4910 & 2.4317 & 3.3815 \\
\hline \hline \multirow{3}{*}{90} & 1 & 2.5225 & 3.5049 & 4.4015 \\
\cline { 2 - 5 } & 2 & 1.3996 & 2.4512 & 3.5385 \\
\cline { 2 - 5 } & 3 & 1.9894 & 3.3514 & 4.4260 \\
\cline { 2 - 5 } & 1 & 3.3340 & 4.9302 & 6.0126 \\
\cline { 2 - 5 } & 3 & 2.0013 & 3.6100 & 5.1632 \\
\hline \hline
\end{tabular}

The table shows the skewness of the cumulative cost for hedging put options with different strike prices and number of periods per rebalancing time, by the three methods and in the setup described in Table 1. 
Table 10: Skewness of the incremental risk

\begin{tabular}{|c|c|c|c|c|}
\cline { 3 - 5 } \multicolumn{2}{c|}{} & \multicolumn{3}{c|}{$\begin{array}{c}\text { Number of periods per } \\
\text { rebalancing time }\end{array}$} \\
\hline Strike & Method & 100 & 300 & 600 \\
\hline \hline \multirow{3}{*}{100} & 1 & 1.7522 & 2.2281 & 3.4271 \\
\cline { 2 - 5 } & 2 & 1.4764 & 2.4414 & 3.6322 \\
\cline { 2 - 5 } & 3 & 1.6865 & 2.7503 & 4.0588 \\
\hline \hline \multirow{3}{*}{95} & 1 & 2.2394 & 3.0319 & 4.4015 \\
\cline { 2 - 5 } & 2 & 1.7524 & 2.9765 & 4.7719 \\
\cline { 2 - 5 } & 3 & 1.9347 & 3.2575 & 4.9737 \\
\hline \hline \multirow{3}{*}{90} & 1 & 2.9436 & 4.3488 & 6.0126 \\
\cline { 2 - 5 } & 2 & 2.2774 & 3.9320 & 6.4523 \\
\cline { 2 - 5 } & 3 & 2.4667 & 4.2173 & 6.5235 \\
\hline
\end{tabular}

The table shows the skewness of the incremental risk for hedging put options with different strike prices and number of periods per rebalancing time, by the three methods and in the setup described in Table 1.

We note that the skewness increases as the number of hedging opportunities decreases and has the largest value when the put option is deep out-of-money.

\section{Discrete hedging put-call parity}

In Section 4 we have only analyzed the case of put options. We will see that hedging call options is closely related to the hedging of put options. Suppose that we have computed the optimal holdings $\xi^{p}, \eta^{p}$ in the portfolio for hedging a put option with maturity $T$, discounted strike price $K$ and $M$ hedging opportunities at $0=t_{0}<t_{1}<\ldots<t_{M-1}<t_{M}:=T$. We can derive a relation between these holdings and the corresponding optimal holdings $\xi^{c}, \eta^{c}$ for the call option on the same underlying asset and with the same maturity, strike price and hedging opportunities. Namely, we have the following property:

$$
\left\{\begin{array}{l}
\xi_{k}^{c}=\xi_{k}^{p}+1 \\
\eta_{k}^{c}=\eta_{k}^{p}-K
\end{array}\right.
$$

for all $0 \leq k \leq M-1$. Thus if we know the optimal holdings for the put option, we can compute the optimal holdings for the call, directly, without solving any optimization problems.

Moreover, the discounted values of the portfolios for hedging the put and the call options, $V_{k}^{p}$ and $V_{k}^{c}$, satisfy the following put-call parity relation for all $0 \leq k \leq M$ :

$$
V_{k}^{c}-V_{k}^{p}=X_{k}-K
$$

This is certainly true at time $T$, since:

$$
V_{M}^{c}-V_{M}^{p}=H^{c}-H^{p}=X_{M}-K,
$$

where $H^{c}=\left(X_{M}-K\right)^{+}$and $H^{p}=\left(K-X_{M}\right)^{+}$. 
For any $0 \leq k \leq M-1$, relation (11) follows immediately from (10). Indeed,

$$
V_{k}^{c}-V_{k}^{p}=\left(\xi_{k}^{c}-\xi_{k}^{p}\right) X_{k}+\eta_{k}^{c}-\eta_{k}^{p}=X_{k}-K
$$

Similarly, we can deduce the relation between the cumulative costs for the call and put options:

$$
C_{M}^{c}=C_{M}^{p}+X_{0}-K
$$

Moreover, the incremental costs for the call and put options are equal, that is, for all $k=0,1, \ldots, M-1$ :

$$
C_{k+1}^{c}-C_{k}^{c}=C_{k+1}^{p}-C_{k}^{p}
$$

Using the above relations, we can easily translate the numerical results for hedging put options, presented in Section 4, to the hedging of call options. Piecewise linear local risk-minimization leads to smaller expected total hedging cost and risk for in-the-money and at-the-money call options, while quadratic risk-minimization tends to perform better as the call options are more deeply out-of-money. Moreover, the initial cost of the hedging portfolio for a call option is usually much smaller for the piecewise linear risk minimization than for the other two methods. Indeed, since $C_{0}^{c}-C_{0}^{p}=X_{0}-K$ for all the three methods and the initial cost $C_{0}^{p}$ is smaller for Method 1 than for the other two methods, $C_{0}^{c}$ will also be smaller for Method 1.

Let us now prove relation (10). The proof given below is valid for all the three methods.

Recall that we have $\xi_{M}=0, \eta_{M}=H$, where $H$ is the discounted payoff. For $k \leq M-1$, in order to find the number of shares $\xi_{k}$ and the number of units of bond $\eta_{k}$ at time $t_{k}$, we have to solve an optimization problem of the form:

$$
\min E\left(f\left(C_{k+1}-C_{k}\right) \mid \mathcal{F}_{k}\right)=\min _{\xi_{k}, \eta_{k}} E\left(f\left(\left(\xi_{k+1}-\xi_{k}\right) X_{k+1}+\left(\eta_{k+1}-\eta_{k}\right)\right) \mid \mathcal{F}_{k}\right) .
$$

where $f(x)=x^{2}$ for Method 2 and $f(x)=|x|$ for Methods 1 and 3. In the case of Method 3 we also have the constraint:

$$
E\left(C_{k+1}-C_{k} \mid \mathcal{F}_{k}\right)=0 \Leftrightarrow \eta_{k}=E\left(\left(\xi_{k+1}-\xi_{k}\right) X_{k+1}+\eta_{k+1} \mid \mathcal{F}_{k}\right) .
$$

Let us first show that relation (10) holds at time $t_{M-1}$.

For all $\left(x^{p}, y^{p}\right)$, let

$$
\left\{\begin{array}{l}
x^{c}=x^{p}+1 \\
y^{c}=y^{p}-K .
\end{array}\right.
$$

Then

$$
\begin{aligned}
E\left(f\left(H^{c}-x^{c} X_{M}-y^{c}\right) \mid \mathcal{F}_{M-1}\right) & =E\left(f\left(H^{c}-X_{M}+K-x^{p} X_{M}-y^{p}\right) \mid \mathcal{F}_{M-1}\right) \\
& =E\left(f\left(H^{p}-x^{p} X_{M}-y^{p}\right) \mid \mathcal{F}_{M-1}\right) .
\end{aligned}
$$

Conversely, for all $\left(x^{c}, y^{c}\right)$, consider $\left(x^{p}, y^{p}\right)$, defined by (14). We have again that relations (15) hold. It follows that:

$$
\left.\left.\min _{x, y} E\left(f\left(H^{c}-x X_{M}-y\right)\right) \mid \mathcal{F}_{M-1}\right)=\min _{x, y} E\left(f\left(H^{p}-x X_{M}-y\right)\right) \mid \mathcal{F}_{M-1}\right)
$$

and $\left(x^{p}, y^{p}\right)$ is optimal for the put problem if and only if $\left(x^{c}, y^{c}\right)$ is optimal for the call problem.

We remark also that $\left(x^{p}, y^{p}\right)$ satisfies the constraint (13) if and only if $\left(x^{c}, y^{c}\right)$ satisfies it. We conclude that relation (10) holds at time $t_{M-1}$ for all the three methods.

Suppose now that we have proved relation (10) at time $t_{k+1}$ and we want to argue it holds at time $t_{k}$. The proof follows exactly in the same way as above by keeping in mind that the optimal holdings at time $t_{k+1}$, that is $\left(\xi_{k+1}^{c}, \eta_{k+1}^{c}\right)$ and $\left(\xi_{k+1}^{p}, \eta_{k+1}^{p}\right)$ satisfy (10).

Therefore, (10) holds for all $0 \leq k \leq M-1$. 


\section{Conclusions}

In an incomplete market, the optimal hedging strategy depends on the criterion for measuring the risk. The traditional strategies found in literature are based on quadratic riskminimization. We remark that the performance of the optimal hedging strategies depends on the moneyness of the option. The numerical results presented in the paper illustrate that piecewise linear risk-minimization leads to hedging strategies with smaller expected total hedging cost and incremental risk for out-of-money and at-the-money put options and respectively, in-the-money and at-the-money call options, while quadratic risk-minimization becomes competitive and can provide a marginal improvement as the put options are more deeply in-the-money and the hedging is frequent enough, or, respectively, the call options are more deeply out-of-money with frequent enough rebalancing. The strategies based on piecewise linear risk-minimization have quite different, and often preferable, properties compared to traditional strategies. The distributions of the total hedging cost and risk show that these new strategies have a larger probability of small cost and risk, though they also have a very small probability of larger cost and risk.

Although there is no analytic solution to the piecewise linear local risk-minimization problem, an optimal strategy can be easily computed. In order to compute the hedging strategy for an option with maturity $T$, we generate a binomial tree on the time horizon $[0, T]$ and compute the portfolio holdings in each state of this tree at hedging times. However, in practice, each time we hedge we may have new information on the volatility and drift of the stock. Therefore, a new binomial tree can be computed at any rebalancing time on the remaining time horizon.

In a complete market, there exists a unique self-financing strategy that exactly replicates the option payoff. By no arbitrage considerations, the option price equals the initial cost of the hedging portfolio. Therefore, pricing and hedging are closely related. In incomplete markets, however, options cannot be priced by arbitrage considerations alone. One may be inclined to consider the expected total hedging cost of the optimal hedging strategy as a fair value of the option (in the case of quadratic risk minimization, the expected total hedging cost equals the initial cost of the hedging portfolio, $C_{0}$ ); generally, this does not, however, include any notion of an economic equilibrium. Bertsimas, Kogan, and Lo (2001) emphasize the fact that the option price has to be " the outcome of a market equilibrium in which investor's preferences, budget dynamics, and information structure interact through the imposition of market-clearing conditions, i.e., supply equals demand". This is why we have focused in our paper on the hedging issue. Moreover, as mentioned by Schweizer (2001), local risk-minimization tries to control the riskiness of a strategy as measured by its incremental risk and it is, therefore, by its very nature, a hedging approach.

Our future research will address the theoretical questions related to the uniqueness and convergence of the optimal trading strategy computed by piecewise linear local riskminimization. In addition, we plan to study the piecewise linear total risk-minimization criterion. We have seen that the total risk measure $E\left(\left|C_{M}-C_{0}\right|\right)$ involves $M+1$ variables (the number of shares of stock in the hedging portfolio at each time $t_{0}, \ldots, t_{M-1}$ and its initial cost $\left.C_{0}\right)$. On the other hand, the local risk measure $E\left(\left|C_{k+1}-C_{k}\right| \mid \mathcal{F}_{k}\right)$ refers to only two variables (the number of shares of stock and the number of units of the bond at time $t_{k}$ ). Using, for example, a binomial tree for the stock price, the local risk-minimization can be expressed as a collection of two-variable piecewise linear minimization problems on subtrees and it is very easy to implement a solver for this case. However, it is much more computationally expensive to solve the total risk-minimization problem. Since $C_{M}-$ $C_{0}$ depends on the entire path of the stock price, a direct approach to the total risk- 
minimization problem min $E\left(\left|C_{M}-C_{0}\right|\right)$ would have to consider all the paths in the binomial tree, and therefore exponentially many variables. We are investigating the implementation of an efficient algorithm to compute the optimal trading strategies using this criterion.

Acknowledgements. The authors are grateful to Prof. Philippe Jorion and the anonimous referee for useful hints and comments.

This research was conducted using resources of the Cornell Theory Center, which is supported by Cornell University, New York State, and members of the Corporate Partnership Program.

\section{Appendix}

Let us analyze in detail the implementation of the local risk-minimizing problems (2), (5) and (7), when using an event tree to describe the stock price. Consider the filtration $\left(\mathcal{F}_{k}\right)_{k=0,1, \ldots, M}$, given by $\mathcal{F}_{k}=\sigma\left(X_{j} \mid j \leq k\right)$, the $\sigma$-field generated by the variables $X_{0}, \ldots, X_{k}$. Suppose the stock price over the time horizon $[0, T]$ is modelled using a binomial tree with $N$ periods, but hedging can only take place on $M<N$ dates $0=t_{0}<t_{1}<\ldots<t_{M-1}<$ $N:=t_{M}$. Thus, for all $0 \leq k \leq M$, at time $t_{k}$ there are $n_{k}=t_{k}+1$ possible states for the stock price and given state $j$ at time $t_{k}$, the stock price can only move to $\alpha_{k}=t_{k+1}-t_{k}+1$ possible states at time $t_{k+1}$ :

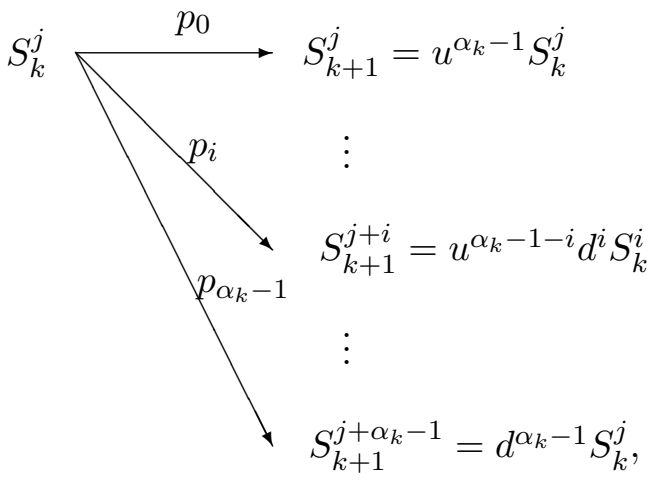

where $p_{i}=\left(\begin{array}{c}\alpha_{k}-1 \\ i\end{array}\right) p^{\alpha_{k}-1-i}(1-p)^{i}$ for all $0 \leq i \leq \alpha_{k}-1$. The parameters $u, d, p$ are defined as follows:

$$
u=e^{\sigma \sqrt{\frac{T}{N}}}, \quad d=\frac{1}{u}, \quad p=\frac{e^{\mu \frac{T}{N}}-d}{u-d}
$$

where $\sigma$ is the volatility of the stock price and $\mu$ its instantaneous expected return.

Recall that the discounted stock price is then given by:

$$
X_{k}^{j}=\frac{S_{k}^{j}}{B_{k}}, \forall 0 \leq k \leq M, \forall 0 \leq j \leq n_{k}-1 .
$$

Suppose now that at time $N$, the discounted payoff of the option in state $j$ is given by $H_{j}$.

Then, the piecewise linear risk minimization problem (5) becomes: starting from $V_{M}=$ $H$, for all the states $j$ at time $t_{k}, k=M-1, \ldots, 0$, minimize

$$
\min E\left(\left|C_{k+1}-C_{k}\right| \mid X_{k}=X_{k}^{j}\right) \text {. }
$$

Therefore: 
- For each $0 \leq j \leq n_{M}-1$ define $\xi_{M}^{j}=0, \eta_{M}^{j}=H_{j}$.

- For each $k=M-1, \ldots, 0$ and for each $0 \leq j \leq n_{k}-1$, find the number of shares $\xi_{k}^{j}$ and the number of units of the bond $\eta_{k}^{j}$ at time $t_{k}$ if state $j$ occurs by solving the minimization problem:

$$
\min _{\xi_{k}^{j}, \eta_{k}^{j}} \sum_{l=0}^{\alpha_{k}-1} p_{l}\left|X_{k+1}^{j+l}\left(\xi_{k+1}^{j+l}-\xi_{k}^{j}\right)+\left(\eta_{k+1}^{j+l}-\eta_{k}^{j}\right)\right| .
$$

If we want to express (17) in a more compact way, for each $k=M-1, \ldots, 0$ and for each $0 \leq j \leq n_{k}-1$, denote by:

$$
A=\left[\begin{array}{cc}
p_{0} & p_{0} X_{k+1}^{j} \\
\vdots & \vdots \\
p_{\alpha_{k}-1} & p_{\alpha_{k}-1} X_{k+1}^{j+\alpha_{k}-1}
\end{array}\right], b=\left[\begin{array}{c}
p_{0}\left(X_{k+1}^{j} \xi_{k+1}^{j}+\eta_{k+1}^{j}\right) \\
\vdots \\
p_{\alpha_{k}-1}\left(X_{k+1}^{j+\alpha_{k}-1} \xi_{k+1}^{j+\alpha_{k}-1}+\eta_{k+1}^{j+\alpha_{k}-1}\right)
\end{array}\right], x=\left[\begin{array}{c}
\eta_{k}^{j} \\
\xi_{k}^{j}
\end{array}\right]
$$

Then (17) becomes:

$$
\min _{x \in \mathbb{R}^{2}}\|A x-b\|_{1} .
$$

As we mentioned before, when the stock price follows a binomial model, the piecewise linear risk-minimization (5) reduces to solving $L^{1}$-minimization problems of the form (18). In order to solve these two-dimensional $L^{1}$-optimization problems, we have implemented an $L^{1}$-solver similar to the solver for the $L^{1}$-norm fit of a straight line described by Karst (1958) and Sadovski (1974).

The constrained piecewise linear risk-minimization method is implemented in a similar way. Using a binomial model for the stock price, problem (7) becomes: starting from $V_{M}=H$, for all the states $j$ at time $t_{k}, k=M-1, \ldots, 0$, minimize

$$
\left\{\begin{array}{l}
\min E\left(\left|C_{k+1}-C_{k}\right| \mid X_{k}=X_{k}^{j}\right) \\
\text { subject to } E\left(C_{k+1}-C_{k} \mid X_{k}=X_{k}^{j}\right)=0 .
\end{array}\right.
$$

Problem (8) is therefore given by:

- For each $0 \leq j \leq n_{M}-1$ define $\xi_{M}^{j}=0, \eta_{M}^{j}=H_{j}$.

- For each $k=M-1, \ldots, 0$ and for each $0 \leq j \leq n_{k}-1$, find the number of shares $\xi_{k}^{j}$ at time $t_{k}$ if state $j$ occurs by solving the minimization problem:

$$
\min _{\xi_{k}^{j}} \sum_{l=0}^{\alpha_{k}-1} p_{l}\left|X_{k+1}^{j+l} \xi_{k+1}^{j+l}-\sum_{s=0}^{\alpha_{k}-1} p_{s}\left(X_{k+1}^{j+s} \xi_{k+1}^{j+s}+\eta_{k+1}^{j+s}\right)-\xi_{k}^{j}\left(X_{k+1}^{j+l}-\sum_{s=0}^{\alpha_{k}-1} p_{s} X_{k+1}^{j+s}\right)\right|
$$

then define: $\eta_{k}^{j}=\sum_{l=0}^{\alpha_{k}-1} p_{l}\left(\xi_{k+1}^{j+l} X_{k+1}^{j+l}+\eta_{k+1}^{j+l}-\xi_{k}^{j} X_{k+1}^{j+l}\right)$.

We can also express this in a more compact way similar to problem (17). For each $k=M-1, \ldots, 0$ and for each $0 \leq j \leq n_{k}-1$, consider the matrices $A$ and $b$ defined before. Denote by $A^{(1)}, A^{(2)}$ the columns of the matrix $A$. Consider also the vector $e=[1, \ldots, 1]^{T}$. Then (19) can be expressed as: 


$$
\left\{\begin{array}{l}
\min _{x \in \mathbb{R}^{2}}\|A x-b\|_{1} \\
\text { subject to: }\left[1, e^{T} A^{(2)}\right] x=e^{T} b
\end{array}\right.
$$

with $x=\left[\begin{array}{l}\eta_{k}^{j} \\ \xi_{k}^{j}\end{array}\right]$.

The above problem can be transformed in a one dimensional minimization problem by substituting $\eta_{k}^{j}$ :

$$
\begin{gathered}
\min _{x \in \mathbb{R}}\left\|\left(A^{(2)}-\left(e^{T} A^{(2)}\right) A^{(1)}\right) x-b+\left(e^{T} b\right) A^{(1)}\right\|_{1} \\
\left\{\begin{array}{l}
\xi_{k}^{j}=x \\
\eta_{k}^{j}=e^{T} b-\left(e^{T} A^{(2)}\right) x .
\end{array}\right.
\end{gathered}
$$

Therefore, the constrained piecewise linear risk-minimization problem (7) reduces to solving one-dimensional $L^{1}$-minimization problems of the form (22) for which a solver is very easily implemented.

Finally, in the framework of the binomial model for the stock price, the quadratic local risk-minimization problem (2) becomes: starting from $V_{M}=H$, for all the states $j$ at time $t_{k}, k=M-1, \ldots, 0$, minimize

$$
\min E\left(\left(C_{k+1}-C_{k}\right)^{2} \mid X_{k}=X_{k}^{j}\right) .
$$

The explicit hedging strategy solving this problem is given by:

- For each $0 \leq j \leq n_{M}-1$ define $\xi_{M}^{j}=0, \eta_{M}^{j}=H_{j}$.

- For each $k=M-1, \ldots, 0$ and for each $0 \leq j \leq n_{k}-1$ define

$$
\left\{\begin{array}{l}
\xi_{k}=\frac{\operatorname{Cov}\left(\xi_{k+1} X_{k+1}+\eta_{k+1}, X_{k+1} \mid X_{k}=X_{k}^{j}\right)}{\operatorname{Var}\left(X_{k+1} \mid X_{k}=X_{k}^{j}\right)} \\
\eta_{k}=E\left(\left(\xi_{k+1}-\xi_{k}\right) X_{k+1}+\eta_{k+1} \mid X_{k}=X_{k}^{j}\right)
\end{array}\right.
$$

where

$$
\begin{gathered}
\operatorname{Cov}\left(\xi_{k+1} X_{k+1}+\eta_{k+1}, X_{k+1} \mid X_{k}=X_{k}^{j}\right)=\sum_{l=0}^{\alpha_{k}-1} p_{l}\left(\xi_{k+1}^{j+l} X_{k+1}^{j+l}+\eta_{k+1}^{j+l}\right) X_{k+1}^{j+l}- \\
\left(\sum_{l=0}^{\alpha_{k}-1} p_{l}\left(\xi_{k+1}^{j+l} X_{k+1}^{j+l}+\eta_{k+1}^{j+l}\right)\right)\left(\sum_{l=0}^{\alpha_{k}-1} p_{l} X_{k+1}^{j+l}\right), \\
\operatorname{Var}\left(X_{k+1} \mid X_{k}=X_{k}^{j}\right)=\sum_{l=0}^{\alpha_{k}-1} p_{l}\left(X_{k+1}^{j+l}\right)^{2}-\left(\sum_{l=0}^{\alpha_{k}-1} p_{l} X_{k+1}^{j+l}\right)^{2}
\end{gathered}
$$

and

$$
E\left(\left(\xi_{k+1}-\xi_{k}\right) X_{k+1}+\eta_{k+1} \mid X_{k}=X_{k}^{j}\right)=\sum_{l=0}^{\alpha_{k}-1} p_{l}\left(\xi_{k+1}^{j+l} X_{k+1}^{j+l}+\eta_{k+1}^{j+l}-\xi_{k}^{j} X_{k+1}^{j+l}\right)
$$




\section{References}

Bertsimas, D., Kogan, L., and Lo, A. (2001). Hedging derivative securities and incomplete markets: An $\epsilon$-arbitrage approach. Operations Research, 49, 372-397.

Föllmer, H., and Schweizer, M. (1989). Hedging by sequential regression: An introduction to the mathematics of option trading. The ASTIN Bulletin, 1, 147-160.

Heath, D., Platen, E., and Schweizer, M. (2001a). A comparison of two quadratic approaches to hedging in incomplete markets. Mathematical Finance, 11, 385-413.

Heath, D., Platen, E., and Schweizer, M. (2001b). Numerical comparison of local riskminimisation and mean-variance hedging. In: Option pricing, interest rates and risk management (ed. E. Jouini, J. Cvitanic and, M. Musiela), pp. 509-537. Cambridge Univ. Press.

Karst, O. (1958). Linear curve fitting using least deviations. J. Amer. Statist. Ass., 53, 118132.

Mercurio, F., and Vorst, T. (1996). Option pricing with hedging at fixed trading dates. Applied Mathematical Science, 3, 135-158.

Sadovski, A. (1974). L1-norm fit of a straight line. Appl. Statist., 23(2), 244-248.

Schäl, M. (1994). On quadratic cost criteria for option hedging. Mathematics of Operation Research, 19(1), 121-131.

Schweizer, M. (1995). Variance-optimal hedging in discrete time. Mathematics of Operation Research, 20, 1-32.

Schweizer, M. (2001). A guided tour through quadratic hedging approaches. In: Option pricing, interest rates and risk management (ed. E. Jouini, J. Cvitanic and, M. Musiela), pp. 538-574. Cambridge Univ. Press. 